\title{
Desafios das Diagonais: Um Jogo Casual para o Aprimoramento do Raciocínio Lógico
}

\section{Wilk Oliveira dos Santos ${ }^{12}$, Gustavo Rosalino de Araújo ${ }^{2}$, Célia Cristina Vilela da Silva $^{3}$}

${ }^{1}$ Núcleo de Excelência em Tecnologias Sociais (NEES) - Instituto de Computação (IC) Universidade Federal de Alagoas (UFAL) - Maceió - AL - Brasil

${ }^{2}$ Virtualize Games - Maceió - AL - Brasil

${ }^{3}$ Curso de Licenciatura em Computação - Universidade de Pernambuco (UPE) Garanhuns - PE - Brasil

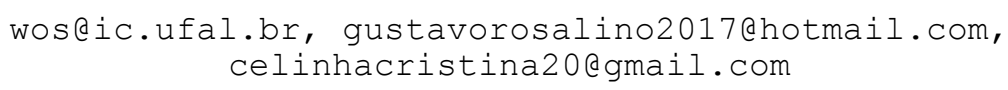

\begin{abstract}
Resumo. É sabido que a disciplina de Matemática é considerada uma das mais complexas do currículo escolar, sendo a principal responsável por reprovações de estudantes do ensino fundamental e médio. Ao mesmo tempo, sabe-se que os jogos representam um importante mecanismo para auxiliar na resolução desta problemática, dado seu caráter lúdico e educacional. Neste sentido, este artigo apresenta o jogo Desafios das Diagonais, um jogo casual para o ensino de conceitos específicos da Matemática. O texto apresenta ainda seu processo de desenvolvimento, por meio do processo de Virtualização de Jogos Educativos.
\end{abstract}

\begin{abstract}
It is well known that the mathematic course is considered one of the most complex topics of the school curriculum, being the main responsible for the failures of elementary and high school students. At the same time, it is known that the games represent an important mechanism to help solve this problem, given its playful and educational character. In this sense, this paper presents the game Challenges of Diagonals, a casual game for the teaching of specific concepts of Mathematic. The paper also presents its development process, through the Educational Games Virtualization process.
\end{abstract}

\section{Cenário de Uso}

Nas últimas décadas, a indústria internacional de desenvolvimento de jogos tem tido um aumento substancial, tornando-se uma indústria mundialmente importante. De acordo com um recente relatório da Entertainment Software Association (ESA 2015), apenas nos Estados Unidos, existem cerca de 155 milhões de jogadores, movimentando em 2014 , cerca de US \$22,41, tendo uma expectativa exponencial de crescimento. Além disso, outro interessante estudo recente mostrou que $74 \%$ dos professores do ensino básico Norte Americano usam jogos digitais em sala de aula (Lofgren 2015), permitindo um crescimento considerável da indústria de jogos educacionais nos últimos anos. 
No contexto acadêmico, uma série de estudos recentes tem sido realizada com o intuito de investigar os efeitos dos jogos em diferentes aspectos educacionais (i.e. Anderson et al. (2010), Scoresby e Shelton (2011) Santos et al. (2014) e outros). Estes estudos têm destacado uma série de discussões relacionadas aos efeitos positivos dos jogos na aprendizagem do aluno, tais como: engajamento, motivação e outros; e efeitos negativos, tais como: violência, ausência de interação social e extrema apreciação de "digital" (atividades digitais realizadas por meio de algum tipo de dispositivo tecnológico), em detrimento de atividades tradicionais (físicas / manuais), trazendo a tona, o conceito de virtualização de jogos educativos, que busca criar versões digitais para jogos tradicionais.

Destaca-se inda o fato que por vezes, os jogos ditos educativos são chatos e sem noção, baseados em conceitos pedagógicos na qual não deveriam ser perceptíveis aos jogadores (Meira 2014). Contudo, vários estudos têm apoiado o uso de jogos para o ensino de disciplinas, com o objetivo de usar os aspectos lúdicos dos jogos a fím de suprimir as dificuldades na aprendizagem de disciplinas consideradas complexas, como Matemática, por exemplo, por meio da virtualização de jogos educativos, que criam versões digitais para jogos tradicionais.

Estudos recentes sobre virtualização de jogos educativos (i.e. Santos et al. (2015) e Oliveira e Silva Junior (2015)), têm citado versões digitais para os jogos educativos tradicionais / físicos, visando manter os aspectos pedagógicos e psicológicos do tradicional / físico Jogo, bem como associar estes aspectos aos elementos contemporâneos do projeto do jogo. Assim, estudos recentes mostraram benefícios diferentes dos jogos virtualizados, bem como destacam uma série de discussões relacionadas ao design, aplicação e avaliação de jogos educativos desenvolvidos com base no processo de virtualização de jogos.

Neste sentido, este artigo apresenta o jogo Desafios das Diagonais, um jogo casual para aprimoramento do raciocínio logico. O jogo é composto por 10 fases estruturadas de forma sequencial de acordo com o nível de dificuldade de cada fase. $\mathrm{O}$ jogo foi desenvolvido para dispositivos moveis com sistema operacional Android ou para computadores pessoais com sistema operacional Windows e foi desenvolvido utilizando uma metodologia especifica para a virtualização de jogos educativos.

\section{Desenvolvimento}

O jogo ora apresentado foi desenvolvido por meio da metodologia $\mathrm{EGV}^{\circledR}$, especifica para virtualização de jogos educativos (Santos e Silva Junior 2016). Esta metodologia abrange desde a escolha do jogo tradicional, por meio de um processo especifico de seleção e avaliação do jogo, perpassando pelas fases de prototipação e desenvolvimento, culminando com a avaliação do jogo em cenário real.

Esta metodologia é uma metodologia de "alto nível", desenvolvida para proporcionar uma estrutura geral que englobe as principais fases do processo de virtualização de jogos educativos, começando pela escolha do jogo tradicional, abordando todo o processo de desenvolvimento do jogo e finalizando na liberação e divulgação do jogo, visando auxiliar o profissional ligado ao design de jogos educativos a desenvolver jogos educativos baseados no processo de virtualização dos jogos. A 
VI Congresso Brasileiro de Informática na Educação (CBIE 2017)

Anais dos Workshops do VI Congresso Brasileiro de Informática na Educação (WCBIE 2017)

metodologia é composta de três fases principais: Pré-produção, Produção e Pósprodução. A Figura 1 apresenta uma diagramação geral de metodologia.

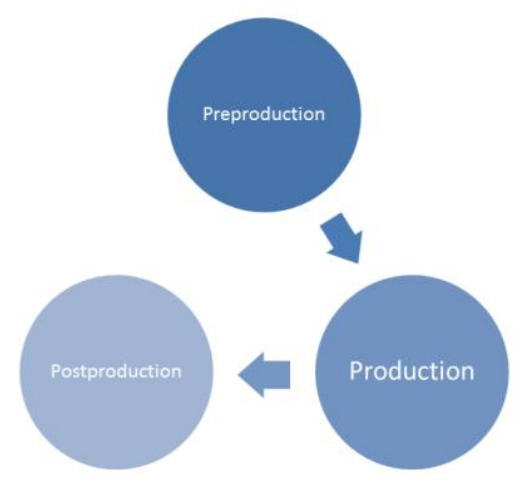

Figura 1: Estrutura geral da metodologia

A fase de pré-produção visa englobar os passos relacionados à escolha do jogo tradicional, análise de requisitos e prototipagem do jogo, a etapa de produção visa cobrir as etapas relacionadas à implementação do jogo, desde a sua documentação até o teste do jogo e a fase de pós-produção, visa abordar a avaliação do jogo, lançamento e manutenção.

A fase de pré-produção é composta por duas etapas gerais: $(i)$ escolha do jogo tradicional responsável pela avaliação de problemas e análise de requisitos (requisitos funcionais e requisitos não funcionais) e (ii) prototipagem, responsável pela prototipagem física e prototipagem virtual do jogo.

A fase de produção é composta por quatro etapas gerais: (i) game design, responsável pela elaboração da documentação do jogo (Game Design Document (GDD)), (ii) projeto, responsável pela modelagem conceitual do jogo, diagramas de fase e projeto de banco de dados, (iii) implementação, responsável pela programação, arte, interface, cenário, personagens, recursos e áudio e (iv) integração, responsável por integrar os componentes e testar a execução e fluxo de fases.

Finalmente, a fase de pós-produção é composta por três etapas gerais: $(i)$ avaliação conceitual, responsável pela avaliação qualitativa, avaliação pedagógica e avaliação psicológica do jogo, (ii) avaliação do projeto, responsável pela avaliação quantitativa (avaliação da usabilidade e avaliação do projeto do jogo) e (iii) implantação, responsável pela liberação e ativação do jogo. 
VI Congresso Brasileiro de Informática na Educação (CBIE 2017)

Anais dos Workshops do VI Congresso Brasileiro de Informática na Educação (WCBIE 2017)

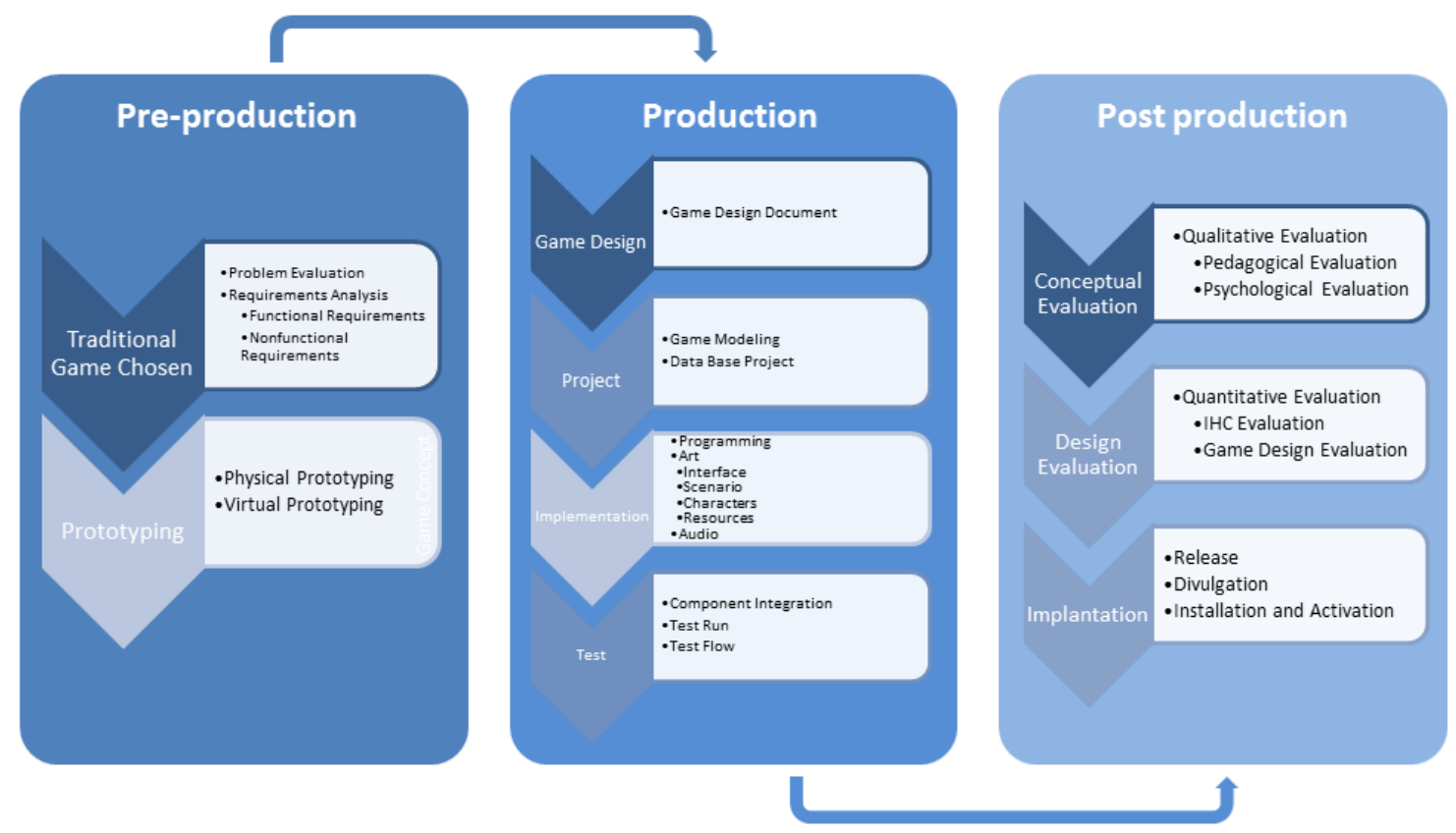

Figura 2: Descrição da Metodologia EGV ${ }^{\circledR}$ (em inglês)

\section{Apresentação do Software}

O jogo Desafio das Diagonais ${ }^{1}$ é composto por 10 fases, organizadas de acordo com seu nível de dificuldade. Em cada uma das fases, o jogador é solicitado a resolver um desafio matemático (implícito no jogo) de raciocínio logico, envolvendo operações básicas e formas geométricas. O jogo é apresentado em três diferentes níveis (fácil, médio ou difícil), apresentando ainda opções de dicas e tutoriais ao longo do jogo.

Ao iniciar o jogo, na primeira tela o jogador tem a opção de verificar o ranking geral do jogo, escolher uma determinada fase ou iniciar o jogo (Figura 3). Caso a escolha jogador seja por verificar as fases e desafios disponíveis, verá uma tela com as opções, sendo que a primeira estará desbloqueada, precisando o jogador resolver o desafio da primeira, para ter acesso a segunda e assim sucessivamente (Figura 4). Finalmente, caso o jogador opte por iniciar o jogo, a primeira vez, verá um tutorial textual explicando como deve proceder durante o jogo (Figura 5).

\footnotetext{
${ }^{1}$ O jogo Desafios das Diagonais é um aplicativo da Empresa Virtualize Games ${ }^{\circledR}$ e pode ser encontrado no seu site oficial (http://virtualizegames.com/), além disso, uma game play do jogo pode ser encontrada no seguinte link: https://youtu.be/mJgbW7hLebw
} 
VI Congresso Brasileiro de Informática na Educação (CBIE 2017)

Anais dos Workshops do VI Congresso Brasileiro de Informática na Educação (WCBIE 2017)

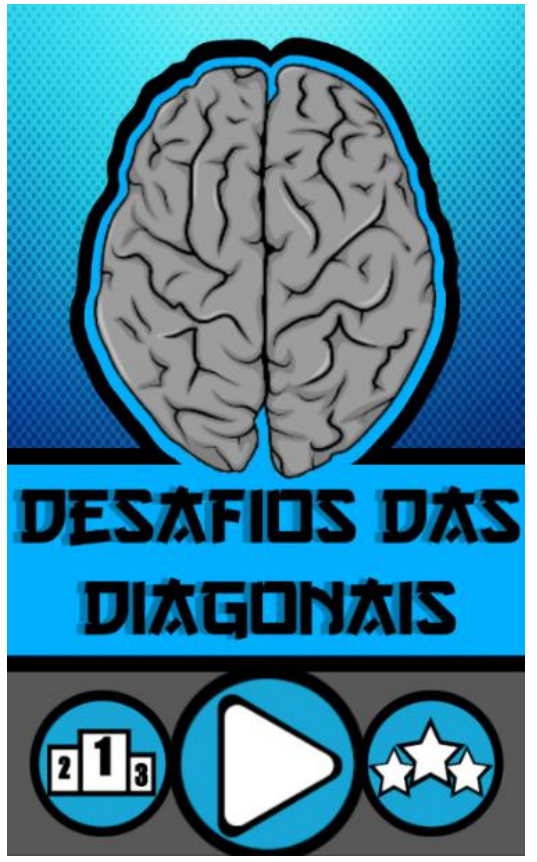

Figura 3: Desafio Das Diagonais - Tela Inicial (versão Beta Mobile)

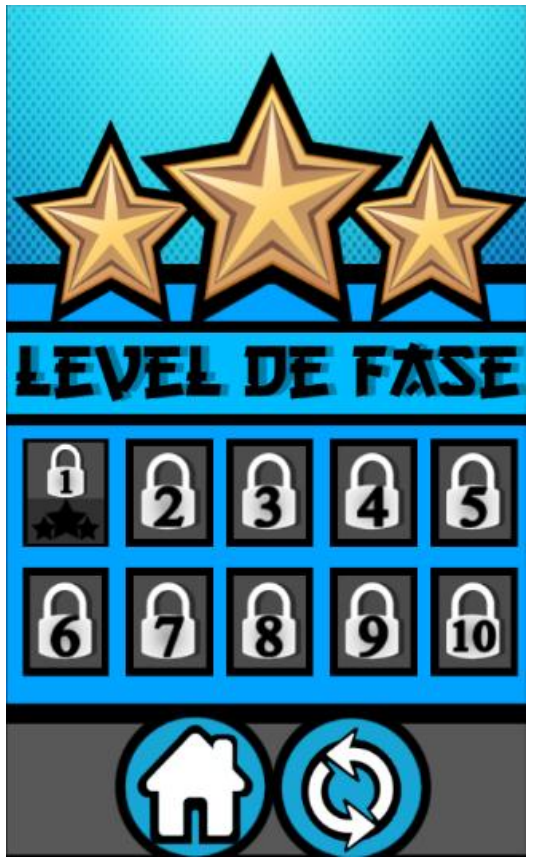

Figura 4: Desafio das Diagonais- Tela de Escolha das Fases (versão Beta Mobile) 
VI Congresso Brasileiro de Informática na Educação (CBIE 2017)

Anais dos Workshops do VI Congresso Brasileiro de Informática na Educação (WCBIE 2017)

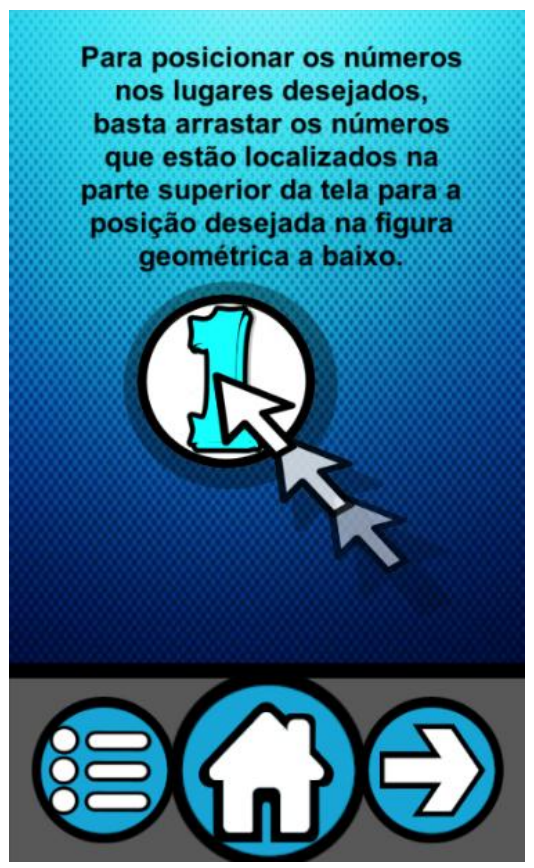

Figura 5: Desafios das Diagonais - Tutorial de Apresentação (versão Beta Mobile)

Ao iniciar uma das fases do jogo, basicamente o jogador verá uma explicação seguida de um tutorial simples, mostrando como o mesmo pode buscar a resolução do desafio. A Figura 6 mostra o tutorial explicativo da primeira fase do jogo e a Figura 7 mostra a fase primeira fase do jogo. Ademais, as fases seguintes seguem a mesma estrutura gráfica e comportamental da primeira ${ }^{2}$.

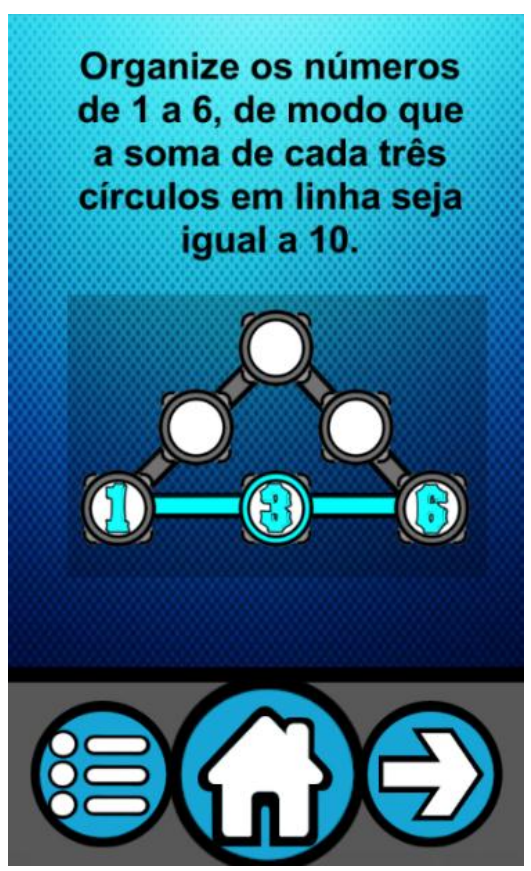

Figura 6: Tutorail da Fase 1

\footnotetext{
${ }^{2}$ Game plays e tutoriais do jogo podem ser encontradas por meio do seguinte link: https://goo.gl/V57Hph
} 


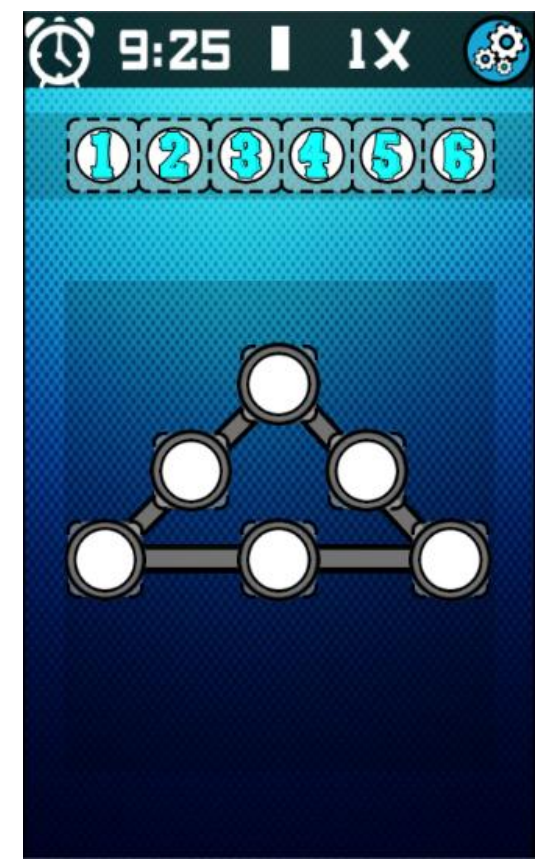

Figura 7: Fase 1

\section{Considerações Finais}

Diante do visível crescimento do uso de diferentes tecnologias em contextos educativos, com destaque para o uso de jogos, bem como, diante dos recentes desafios relacionados ao desenvolvimento e aplicação de jogos para educação, especialmente, os desafios que cernem a criação de versões digitais para jogos digitais outrora usados de forma positiva no ensino de diferentes conceitos (virtualização de jogos), trona-se importante o desenvolvimento de novos jogos com esta finalidade (Santos e Silva Junior 2014).

Neste sentido, este artigo apresentou o jogo Desafios das Diagonais, desenvolvido por meio do processo de virtualização de jogos educativos (Santos e Silva Junior 2016). O jogo apresentado foi desenvolvido por meio da metodologia $E G V^{\circledR}$ uma metodologia especifica para a virtualização de jogos educativos, bem como tem sido avaliado por meio de diferentes métricas, por diferentes grupos de pesquisas nacionais.

Diante do exposto, almeja-se como trabalhos futuros o desenvolvimento de novas fases para o jogo, bem como a sua disponibilização gratuita para dispositivos mobile. Vislumbra-se ainda o desenvolvimento de novos jogos por meio do processo de virtualização de jogos, para diferentes conteúdos de diferentes disciplinas. Recomendase ainda a realização de novos estudos empíricos ligados a virtualização de jogos educativos.

\section{References}

Anderson, C. A., \& Gentile, D. A. (2014). Violent Video Game effects on Aggressive thoughts, feelings, physiology, and Behavior. Media Violence and Children: A Complete Guide for Parents and Professionals, 229.

Essential facts about the computer and video game industry: 2015 sales, demographic and usage data. Entertainment Software Association, 2015. 
VI Congresso Brasileiro de Informática na Educação (CBIE 2017)

Anais dos Workshops do VI Congresso Brasileiro de Informática na Educação (WCBIE 2017)

Lofgren, K. (2015) 2015 Video Game Statistics \& Trends Who's Playing What \& Why?. Available in: http://www.bigfishgames.com/blog/2015-global-video-gamestats-whos-playing-what-and-why/. Accessed: 09/March/2016.

Meira, S., 2014. Cadê os jogos educacionais móveis? [Online] Available at: HYPERLINK "http://boletim.de/silvio/cad-os-jogos-educacionais-mveis/" http://boletim.de/silvio/cad-os-jogos-educacionais-mveis/ Accessed em 9 de Julho de 2014.

Oliveira, W., \& da Silva Junior, C. G. (2015). Pesquisa, Desenvolvimento e Avaliação de um Jogo para o Ensino de Matemática, Baseado no Processo de Virtualização de Jogos. In Anais dos Workshops do Congresso Brasileiro de Informática na Educação (Vol. 4, No. 1, p. 145).

Oliveira, W., Neto, S., da Silva Junoir, C. G., \& Bittencourt, I. I. (2015, October). Avaliação de Jogos Educativos: Uma Abordagem no Ensino de Matemática. In Brazilian Symposium on Computers in Education (Simpósio Brasileiro de Informática na Educação-SBIE) (Vol. 26, No. 1, p. 657).

Santos, W. O., \& da Silva Junior, C. G. (2016) State of the Art in Educational Games Virtualization. RENOTE, 14(1).

Santos, W. O., \& Silva Junior, C. G. (2016). Challenges of Games Virtualization Applied to Educational Games. In V Workshop of Challenges of Computers in Education (pp. 597-606).

Santos, W. O.; da Silva, A. P.; Silva Junior, C. G. (2014) "Conquistando com o Resto: Virtualização de um Jogo para o Ensino de Matemática”. In: Anais do Simpósio Brasileiro de Informática na Educação (SBIE). p. 317-321.

Santos, W. O.; Silva Junior, C. G. (2014) "Uso de Jogos no ensino da Matemática: Uma análise entre os jogos tradicionais e os jogos digitais, baseada em pesquisa e mapeamento dos materiais encontrados na Web”. In: X Seminário Jogos Eletrônicos, Educação e Comunicação. Salvador - BA.

Santos, W., Silva Neto, S. R., \& Silva Junior, C. G. (2013). Uso de Games no ensino da Matemática. Uma proposta de virtualização dos jogos tradicionais, para uso como mecanismo de apoio ao processo de ensino e aprendizagem. In: Anais do Simpósio Hipertexto e Tecnologias na Educação, Recife-PE.

Scoresby, J., \& Shelton, B. E. (2011). Visual perspectives within educational computer games: effects on presence and flow within virtual immersive learning environments. Instructional Science, 39(3), 227-254. 